\title{
Atypical autonomic regulation, auditory processing, and affect recognition in women with HIV
}

\author{
K.J. Heilman ${ }^{1}$, E.R. Harden ${ }^{1}$, K.M. Weber ${ }^{2}$, M. Cohen ${ }^{2,3}$, and S.W. Porges ${ }^{1,4}$ \\ ${ }^{1}$ Brain-Body Center, Department of Psychiatry, University of Illinois at Chicago, Chicago, IL USA \\ 2 The CORE Center/ Cook County Health and Hospital System and Hektoen Institute of Medicine, \\ Chicago, IL USA \\ ${ }^{3}$ Departments of Medicine, Stroger Hospital and Rush University, Chicago, IL USA \\ ${ }^{4}$ Department of Psychiatry, University of North Carolina at Chapel Hill, Chapel Hill, NC USA
}

\begin{abstract}
This study examined the effect of HIV on visceromotor (i.e., heart rate and heart rate variability) and somatomotor (i.e., auditory processing and affect recognition) components of a social engagement system defined by the Polyvagal Theory (Porges, 1995) that links vagal regulation of the heart with brainstem regulation of the striated muscles of the face and head. Relative to at risk HIV- seronegative women, HIV-seropositive women had less heart rate variability (i.e., respiratory sinus arrhythmia) and had poorer performance on auditory processing and affect recognition tasks. CD4 was negatively correlated with the accuracy to detect specific emotions. The observed indices of atypical autonomic and behavioral regulation may contribute to greater difficulties in social behavior and social communication between HIV- infected women and other individuals in their social network.
\end{abstract}

\section{Keywords}

HIV; women; respiratory sinus arrhythmia; heart rate; heart rate variability; auditory processing; affect recognition; Polyvagal Theory

\section{Introduction}

HIV-infected individuals are at risk for various physical and psychiatric illnesses that may negatively impact their social interactions (e.g., Applebaum et al., 2010; Pence et al., 2006; Ciesla and Roberts, 2001). For example, studies suggest that individuals living with HIV are at high risk for depressive symptoms (Lyon and Munro, 2001; Schrimshaw, 2003; Prachakul et al., 2007), which may impede the ability to negotiate stable long-term relationships and social support (Prachakul et al., 2007). We investigated and compared neurophysiological

\footnotetext{
(C) 2013 Elsevier B.V. All rights reserved.

Corresponding author: Stephen W. Porges, PhD Professor Department of Psychiatry University of North Carolina at Chapel Hill 387 Medical School Wing D Campus Box 7160 Chapel Hill, NC 27599-7160 stephen_porges@med.unc.edu. Keri J. Heilman kheilman@psych.uic.edu Emily R. Harden eharden456@gmail.com Kathleen M. Weber kathleen.weber@ hektoen.org Mardge Cohen mardge.cohen@gmail.com.

Publisher's Disclaimer: This is a PDF file of an unedited manuscript that has been accepted for publication. As a service to our customers we are providing this early version of the manuscript. The manuscript will undergo copyediting, typesetting, and review of the resulting proof before it is published in its final citable form. Please note that during the production process errors may be discovered which could affect the content, and all legal disclaimers that apply to the journal pertain.
} 
and biobehavioral features that may contribute to compromised social behavior in HIVinfected and socio-demographically similar at risk uninfected women in Chicago. An understanding of these features may enable HIV clinicians and members of support networks to be more aware of the direct and indirect impact of HIV on social and psychological functioning and adapt communication accordingly.

The Polyvagal Theory (Porges, 1995, 2007, 2009, 2011; Porges and Furman, 2011) provides a theoretical framework to investigate the impact of HIV on the neural regulation of the autonomic nervous system, auditory processing, and social behavior. The theory describes the integration of the neural regulation of the heart, via the vagus, with the neural regulation of the striated muscles of the face and head, including the neural regulation of middle ear muscles, via other cranial nerves. This heart-face connection operates as an adaptive and functional Social Engagement System during face-to-face interactions. The Social Engagement System is a convergence of neural mechanisms, with synergistic visceromotor and somatomotor components, that interact with ingestion, state regulation and social engagement processes (Porges, 2007) to support social behavior. The system incorporates a visceromotor component that regulates the heart and bronchi via a myelinated branch of the vagus and a somatomotor component that regulates the striated muscles of the face and head via the special visceral efferent neural pathways traveling through several cranial nerves. The somatomotor component regulates several muscles with specific social engagement functions, namely (1) middle ear muscles via cranial nerves V and VII to extract human voice from backgrounds sounds; (2) laryngeal and pharyngeal muscles via cranial nerves IX and $\mathrm{X}$ to express affect in vocalizations; (3) muscles of mastication for ingestion via cranial nerve V; (4) muscles of the face for emotional expression via cranial nerve VII; and (5) muscles that tilt the head for gesturing via cranial nerve XI. Studies have shown support for a down-regulated Social Engagement System in populations with difficulties in social behavior. Indicators of a down-regulated Social Engagement System may include difficulties with state regulation, auditory processing, prosody, and/or eye gaze. For example, several studies have reported atypical vagal regulation, indexed via respiratory sinus arrhythmia (RSA) (i.e., the high-frequency component of heart rate variability within the frequency of spontaneous breathing), in clinical disorders characterized by atypical social behavior, such as autism spectrum disorder (Patriquin et al., 2013; Daluwatte et al., 2012) and fragile $X$ syndrome (Hall et al., 2009; Roberts et al., 2001) and in children at risk for the development of externalizing/internalizing behavioral problems (Calkins et al., 2007).

As proposed by the Polyvagal Theory, if HIV is associated with a down-regulated Social Engagement System, HIV- infected individuals may have difficulty detecting positive emotional states of partners, family members, caregivers and other individuals involved in their social support. Thus, in understanding the social dynamics of care and support of HIVinfected women, it is important to evaluate the features of the Social Engagement System.

Several factors related to HIV infection may lead to down regulation of the Social Engagement System. First, HIV may affect the neural (vagal) regulation of the heart reflected in measures of heart rate and heart rate variability including respiratory sinus arrhythmia (RSA). Several researchers have reported that individuals with HIV had faster heart rates (Cade et al., 2007; Lebech et al., 2007; Mittal et al., 2004) and less heart rate variability (Mittal et al., 2004). However, other studies have not supported these findings and have reported that HIV-infected individuals did not have significantly faster heart rate (Correia et al., 2006; Sakhuja et al., 2007; Brownley et al., 2001) or less heart rate variability (Lebech et al., 2007; Correia et al., 2006; Sakhuja et al., 2007; Brownley et al., 2001). 
HIV disease progression may impact directly on central processes as well as the neural pathways of both the visceromotor (i.e., vagal) and somatomotor (i.e., neural regulation of the striated muscles of the face and head) components of the Social Engagement System. Cognitive deficits associated with the HIV virus and/or treatment are common (e.g., Weber et al., 2013; Robertson et al., 2007). Deficits in central cholinergic function have been studied as a potential mechanism for cognitive deficits (Martinez-Ruiz et al., 2000; Marubio and Paylor, 2004). Pharmacologic manipulations dampening central cholinergic function with drugs such as scopalomine have degraded performance on dichotic listening tasks (Drachman et al., 1980). Similarly peripheral function dependent on cholinergic systems (e.g., parasympathetic tone, middle ear muscle function) also reflects indices of a deficit. A negative relation has been reported (Compstolla et al., 2008) between HIV disease progression [depletion of CD4+ T cells (CD4 hereafter)] and heart rate. Moreover, since HIV is neurotoxic, it may result in peripheral nerve damage (Simpson, 1994) including damage to cranial nerves regulating the striated muscles of the face and head. Consistent with this hypothetical pathway, there is a report (Komolafe et al., 2009) that the extra-axial segment of the facial nerve is typically involved in the early stages of HIV infection. Since the facial nerve also innervates the muscles of the middle ear, damage to the facial nerve might also affect auditory processing making it difficult to extract human speech from background noise.

The current study assessed the degrading effects of HIV on both the functioning of the visceromotor component (i.e., RSA) and the functioning of the somatomotor component (i.e., auditory processing and affect recognition) of the Social Engagement System. The study tested the following four hypotheses (1) HIV-infected women will have dampened vagal regulation of the heart, reflected in faster resting heart rate and lower amplitude RSA; (2) HIV-infected women will have auditory processing difficulties and perform poorer on a dichotic listening task; (3) HIV-infected women will have affect recognition difficulties and make more errors and respond slower on an affect recognition task; and (4) virologic and immunologic indices of HIV disease progression will be related to heart rate, RSA, auditory processing and affect recognition.

\section{Methods}

\subsection{Participants}

This investigation was conducted between February 2006- February 2009 at the Chicago site of the NIH funded Women's Interagency HIV Study (WIHS), an ongoing cohort study of the treated history of HIV infection and related health conditions among HIV-seropositive and at-risk demographically similar women who were HIV- seronegative. Recruitment, retention, protocols, procedures, and demographics of the WIHS have been described elsewhere (Barkan et al., 1998; Hessol et al., 2009). Enrollment began in 1994 with an expansion during 2001-2002 (Bacon et al., 2005). HIV serostatus was determined by Elisa and confirmed by Western blot at study entry for all participants and semiannually thereafter for those initially seronegative. WIHS study participants are interviewed twice yearly about medical history and behaviors and undergo an examination and collection of study specimens to determine current clinical status. Chicago participants were recruited and evaluated in the experimental protocol if they were between ages 25-60 years and were English-speakers and excluded if they were unable to abstain from using illicit substances for 24 hours prior to the research session, had a confirmed psychiatric diagnosis of psychosis, had a chronic medical condition (other than HIV infection) that was not controlled with medications (current high blood pressure or high blood sugar), showed evidence of current HCV viremia had medically documented auditory or visual impairment, had a documented heart condition and/or history of stroke, or were currently pregnant, post- 
partum or lactating. Written informed consent was obtained after local human subjects' committee approval.

Seventy-three HIV- seropositive (HIV-SP) and 25 HIV- seronegative (HIV-SN) women were enrolled. The ratio of HIV- seropositive to HIV- seronegative participants in the study reflected the serostatus ratio of individuals who were enrolled in the Chicago WIHS cohort.

\subsection{Apparatus and materials}

2.2.1 Physiological measures-Heart rate and RSA were recorded from all participants during the experimental protocol. A LifeShirt ${ }^{\circledR}$ (Vivometrics) was used to collect cardiac data and respiration rate. The LifeShirt ${ }^{\circ}$ has been benchmarked against the Biopac system and provides measures with sufficient accuracy to generate sensitive measures of heart rate and RSA (see Heilman and Porges, 2007). For all participants, three $\mathrm{Ag} / \mathrm{AgCl}$ self-adhering electrodes (Conmed Corp., Utica, NY) with a contact area of $10 \mathrm{~mm}$ diameter were placed directly onto the upper chest and on the lateral surface of the abdomen for data collection.

2.2.2 Cognitive measure-The Wechsler Test of Adult Reading (WTAR; Wechsler 2001) was used as a measure of intellectual functioning and has been co-normed with the Wechsler Adult Intelligence Scale.

2.2.3 Immunologic and virologic indices of HIV disease progression-Immune function parameters and HIV viral load were obtained from labs certified by the NIH Viral and Immunology Quality Assurance Programs. Analytic approaches using these measures were previously described in Cole, Hernan, Anastos, Jamieson \& Robins (2007). Flow cytometry was performed at NIH ACTG-certified laboratories. HIV viral load was measured using a lower level of detection of 40 copies per ml. HIV viral load was $\log 10$ transformed to normalize the distribution.

\subsection{Procedures}

Participant consent was obtained prior to the start of the study. After obtaining consent, the physiological monitoring equipment was demonstrated to the participant, the ECG electrodes were placed and the study protocol initiated. All protocol and task instructions were presented verbally. Due to the consent process, paperwork and equipment placement, there was an approximate time lapse of 30 minutes between arrival to the study and the physiological baseline recording. The protocol consisted of several sequential components (1) a physiological baseline recording during which the participant was encouraged to sit as quietly as possible and to minimize motor activity for 3 minutes (i.e., "initial physiological baseline"); (2) the measurement of auditory processing skills using two subscales (filtered words and competing words) from the SCAN (The Psychological Corporation, San Antonio, TX) developed by Keith (1986); (3) a second, 3-minute physiological baseline recording (i.e., "pre-DARE baseline"); (4) a test of affect recognition, Dynamic Affect Recognition Evaluation (DARE; Porges et al., 2007); and (5) a final, 3-minute post-protocol physiological recording baseline (i.e., "post-DARE baseline").

Two tests, the Filtered Words Test and the Competing Words Test, from the SCAN were used to evaluate auditory processing. These tests require low task demands and require only that the participant repeat the stimulus word or words. The Filtered Words Test assesses the ability to identify words that are muffled (i.e., low passed) and the Competing Words Test assesses the ability to accurately report the word being presented to the left or right ear when two different words are simultaneously presented to the left and right ears. As proposed by the Polyvagal Theory (Porges and Lewis, 2009), performance on the Filtered Words test would be dependent on the transfer function of the middle ear structures to dampen the 
influence of the competing low frequency sounds on the participant's ability to identify words that have been low passed. Thus, performance on the Filtered Words task would be a functional index of the Social Engagement System and hypothetically should parallel RSA. While it is proposed that performance on the Filtered Words test would parallel peripheral "cholinergic" regulation of the heart (i.e., RSA), the Competing Words test involves a more complex dependence on central "cholinergic" pathways that are related to cognitive function. Thus, individual differences in Competing Words performance may be more sensitive to cognitive function (i.e., IQ) and less sensitive to the neural regulation of peripheral autonomic state (i.e., RSA). The duration of the SCAN was approximately 10 minutes.

The Dynamic Affect Recognition Evaluation (DARE) is a standardized tool for assessing facial emotion recognition (Porges et al., 2007; Bal et al., 2010). DARE uses the CohnKanade Action Unit-Coded Facial Expression Database (Cohn et al., 1999) to create video sequences of facial expressions. The stimuli included uncompressed video files (i.e., series of still images) consisting of six basic emotions. The images were morphed and the final videos included a face starting with a neutral expression and slowly transitioning into one of the six target emotions (Sad, Fear, Surprise, Disgust, Anger, and Happiness). Video durations ranged from 15 to 33 seconds, depending on the number of the frames in the original image sequences, which was independent of emotion category. Participants were asked to indicate when they could identify the emotion (latency) and then verbally identify the emotion (accuracy). Duration of DARE administration ranged from 10-20 minutes, depending on the average latency of each individual participant to respond to the stimuli.

2.3.1 Data reduction-Heart rate data were visually inspected and edited off-line with CardioEdit software (Brain-Body Center, University of Illinois at Chicago). Editing consisted of integer arithmetic (i.e., dividing intervals when detections were missed and adding intervals when spuriously invalid detections occurred) or manual insertion/deletion of missing/spurious detections based on the ECG recording. No more than 5\% of the data in each file required editing.

RSA (i.e., the high-frequency component of heart rate variability) defined as variance within the frequency band associated with spontaneous breathing, was calculated with CardioBatch software (Brain-Body Center, University of Illinois at Chicago) consistent with the procedures developed by Porges (1985). CardioBatch quantifies the amplitude of RSA using age-specific parameters that are sensitive to the maturational shifts in the frequency of spontaneous breathing. The method when applied to adult participants includes the following steps (1) timing sequential R-R intervals to the nearest millisecond; (2) producing time-base data by resampling the sequential R-R intervals into 500 msec intervals; (3) detrending the time-based series with a 21-point cubic moving polynomial (Porges and Bohrer, 1990) that is stepped through the data to create a smoothed template and the template is subtracted from the original time-based series to generate a detrended residual series; (4) bandpass filtering the detrended time series to extract the variance in the heart period pattern associated with spontaneous breathing in adults $(.12-.40 \mathrm{~Hz})$; and (5) transforming the variance estimates with a natural logarithm to normalize the distribution of RSA estimates (Riniolo and Porges, 1997). These procedures are statistically equivalent to frequency domain methods (i.e., spectral analysis) for the calculation of the amplitude of RSA when heart period data are stationary (Porges and Byrne, 1992). RSA is an accurate non-invasive index of the vagal influence to the heart being mediated via myelinated vagal pathways that originate in the brainstem nucleus ambiguus (see Lewis et al, 2012; Porges, 2007). 
Three minutes of cardiac data during each baseline condition were analyzed for heart rate and RSA. Each cardiac variable was quantified during sequential 30-sec epochs within each of the three minute baselines and averages of the within condition epochs were used in the data analyses. Median respiration rate was calculated for the same durations and conditions as the cardiac data.

2.3.2 Data/Participant loss-Participants were asked to provide information regarding recent use of illegal drugs, medications and tobacco upon arrival for the research study. Four participants were prematurely consented to participate in the study prior to providing this information, and no data were collected for these participants who were immediately excluded due to admitted use of crack, cocaine, heroin and/or marijuana within the two days prior to the research session (3 HIV-SP, 1 HIV-SN). Additionally, two participants who were taking over-the-counter and/or prescribed medications that interfere with autonomic functioning were also excluded from the protocol (1 HIV-SP took NyQuil, 1 HIV-SP took Depakote).

Participants were not asked to refrain from tobacco products prior to the study, nor were they excluded for use of tobacco products. One participant reported tobacco intake within 2-3 hours prior to the research session (1 HIV-SP), seven participants reported tobacco intake within1- 2 hours prior to the research session (6 HIV-SP, 1 HIV-SN) and thirteen participants reported tobacco intake within 1 hour prior to the research session (7 HIV-SP, 6 HIV-SN). However, because tobacco use during the 3 hours prior to the study did not have a consistent effect on baseline physiological measures, possibly due to the 30 minute time lapse between arrival for the study and the first physiological recording (during which, the participants were not allowed tobacco), the data from these participants were included in the data analyses.

Participants were asked to refrain from alcohol use for 12 hours prior to arriving for the research study. All participants reported compliance with this request.

Participants were also asked to refrain from caffeine use for 2 hours prior to arriving for the research study, but were not excluded from the study for caffeine consumption. Six participants reported consuming caffeine 2 hours prior to the research session (4 HIV-SP, 2 HIV-SN), and four participants reported consuming caffeine 1 hour prior to the research session (2 HIV-SP, 2 HIV-SN). Two participants reported consuming caffeine immediately prior to the research session (1 HIV-SP, 1 HIV-SN). Because caffeine consumption during the 2 hours prior to the study did not have a consistent effect on baseline physiological measures, possibly due to the 30 minute time lapse between arrival for the study and the first physiological recording (during which, the participants were not allowed caffeine), the data from these participants were included in the data analyses.

Participants were also asked to report any history of cardiovascular disease or stroke. One participant reported cardiovascular disease, and her data were excluded from analyses (1 HIV-SP).

Complete data from eight additional participants were excluded after beginning the research protocol, due to equipment failure (5 HIV-SP, $1 \mathrm{HIV}-\mathrm{SN}$ ) or drowsiness/sleeping during research session (1 HIV-SP, 1 HIV-SN).

Table 1 lists demographic and clinical characteristics of the included study participants by HIV serostatus, including any statistical differences between groups. 
2.3.3 Statistical analyses-There were no group differences in age, BMI, or WTAR. Age was significantly correlated with RSA, $r(80)=-.39, p<.000$. Age was not used as a covariate in the RSA analyses, since there were no group differences in age and the correlations between age and RSA were of similar magnitude and direction within each group. Age was not correlated with respiration rate, heart rate, or performance on the SCAN subtests or the DARE. Because of the potential effect of body mass index (BMI) on the physiological measures, the correlation between BMI and each physiological variable was calculated. BMI was not correlated with any of the physiological variables.

Group differences in initial autonomic activity were evaluated using separate analyses of variance (ANOVA) for each dependent variable (Group = HIV serostatus; Dependent variables $=$ initial baseline RSA, heart rate, respiration rate). Change in baseline variables during the course of the protocol were evaluated using repeated-measures ANOVA for each dependent variable (Group = HIV serostatus; Dependent variables = initial baseline, preDARE baseline, post-DARE baseline for RSA, heart rate, respiration rate).

Group differences in auditory processing (Filtered Words, Competing Words) were evaluated using separate ANOVAs for each subtest (Group = HIV serostatus; Dependent variables $=$ Filtered Words standard score, Competing Words standard score) .

Group differences in accuracy on the DARE were evaluated using Mann-Whitney U test (Group = HIV serostatus; Dependent variables $=$ total number of errors for Sad, Fear, Surprise, Disgust, Anger, Happiness videos). Group differences in response latency on the DARE were evaluated using MANOVA (Group = HIV serostatus; Dependent variables = average response latency for Sad, Fear, Surprise, Disgust, Anger, Happiness videos).

Correlations were calculated to evaluate the relationships between accuracy and response latency, and between WTAR levels and both accuracy and latency. In addition, correlations were calculated to evaluate the relationships between physiological baselines and performance on SCAN and DARE (accuracy and latency), between viral load (CD4) and both the behavioral measures (SCAN; DARE accuracy, latency), and the physiological baselines (RSA, heart rate, and respiration rate). Correlations were also used to evaluate the effect of duration of HIV infection on physiological baselines and behavioral measures.

\section{Results}

\subsection{Initial baseline variables}

Descriptive statistics (mean, standard deviation) for each physiological variable, during each baseline, are presented in Table 2.

Analyses of variance (Group = HIV serostatus; Dependent variables $=$ initial baseline RSA, heart rate, respiration rate) ${ }^{1}$ demonstrated that RSA was significantly lower in the HIV-SP group, $F(1,79)=5.61, p<.02\left(\eta \rho^{2}=.07\right.$, observed power $\left.=.65\right)$. There were no significant group differences, by serostatus, for initial baseline heart rate or respiration rate.

\subsection{Change in physiological data across protocol}

Repeated-measures ANOVA evaluated group differences across the three baselines (Group = HIV serostatus; Dependent variables = initial baseline, pre-DARE baseline, post-DARE baseline for RSA, heart rate, and respiration rate). ${ }^{2}$ Although there were no group or interaction effects, across the three baseline conditions there were significant changes in

\footnotetext{
${ }^{1}$ HIV-SP $n=60$, HIV-SN $n=21$. Data loss from 2 participants was due to equipment problems (1 HIV-SP; 1 HIV-SN).
} 
heart rate, $F(1.64,109.86)=47.61, p<.000\left(\eta \rho^{2}=.42\right.$, observed power $\left.=1.00\right)$, and RSA, $F(1.60,107.15)=7.35, p<.002\left(\eta \rho^{2}=.10\right.$, observed power $\left.=.89\right)$, as heart rate significantly decreased and RSA significantly increased as the study progressed. There were no condition, group or interaction effects for respiration rate.

\subsection{Auditory processing performance}

Univariate ANOVA (Group $=$ HIV serostatus; Dependent variables $=$ Filtered Words Standard Score, Competing Words Standard Score) demonstrated that the HIV-SP group performed significantly worse on the Competing Words test, $F(1,81)=5.60, p<.02\left(\eta \rho^{2}=\right.$. 07 , observed power $=.65)$ (see Figure 1$)$. Although the direction of responding was similar on the Filtered Words test [Mean $(\mathrm{HIV}-\mathrm{SN})=9.45$, Mean $(\mathrm{HIV}-\mathrm{SP})=8.47$ ], the group differences were not statistically significant, $F(1,81)=1.85, p<.18, \eta \rho^{2}=.023$, observed power $=.27($ see Figure 1$)$.

\subsection{Dynamic Affect Recognition Evaluate (DARE) performance}

Seventy-six participants were included in the DARE accuracy and latency analyses. Seven participants did not have complete data for the DARE due to equipment problems and/or incomplete testing (6 HIV-SP, 1 HIV-SN).

3.4.1 Accuracy-Non-parametric Mann-Whitney U test (Group = HIV serostatus; Dependent variables $=$ total number of errors for Sad, Fear, Surprise, Disgust, Anger, Happiness videos) demonstrated that the HIV-SP group made significantly more total errors across all emotions, ( $\mathrm{U}$ test, $p<.03$ ). There were no group differences within any particular emotion category.

3.4.2 Latency-MANOVA (Group = HIV serostatus; Dependent variables = average latency for Sad, Fear, Surprise, Disgust, Anger, Happiness videos) demonstrated that there were no significant group differences in latency across all emotions or for any specific emotion.

Correlational analyses were conducted to evaluate relationships between accuracy and latency. When collapsed across groups, accuracy and latency were negatively correlated (i.e., shorter latencies with more error responses) for Fear videos, $r(76)=-.29, p<.01$. Within the HIV-SN group, accuracy and latency were correlated for Fear videos, $r(21)=-$. $45, p<.04$. Additionally, accuracy and latency were negatively correlated when averaged across all videos, $r(21)=-.48, p<.03$. Accuracy and latency were not significantly correlated within the HIV-SP group for any emotion.

3.4.3 Accuracy, latency and WTAR scores-Although not statistically significant, there was a trend towards lower WTAR performance in the HIV-SP group, $F(1,75)=3.02$, $p<.09$. Moreover, within the HIV-SP group, WTAR was significantly correlated with Competing Words performance, $r(53)=.29, p<.04$.

Correlations were calculated to determine whether accuracy or latency was related to WTAR scores within each emotion. Seventy participants had both WTAR and DARE data (accuracy and latency) available for correlational analyses, as seven participants (7 HIV-SP) attempted the WTAR assessment, but became frustrated and refused to complete the assessment, rendering the score invalid for analyses.

\footnotetext{
${ }^{2}$ For analyses of heart rate and RSA, HIV-SP $n=50$, HIV-SN n=19. Data loss from 14 participants due to equipment problems (10 HIV-SP, 2 HIV-SN) and participant withdrawal during the protocol (1 HIV-SP). For analyses of respiration rate, HIV-SP n=50, HIV$\mathrm{SN} \mathrm{n}=19$.
} 
When collapsed across groups, WTAR scores were negatively correlated with total number of errors for the Sad videos, $r(70)=-.26, p<.03$, total number of errors for the Disgust videos, $r(70)=-.28, p<.02$, and total number of errors for the Anger videos, $r(70)=-.45, p<$. 000 . Additionally, WTAR scores were negatively correlated with the total number of errors across all of the videos, $r(70)=-.36, p<.002$. The findings indicate that the lower the WTAR score, the greater number of total errors, and greater number of errors specifically on the Sad, Disgust and Anger videos.

Within the HIV-SN group, WTAR scores were not correlated with any accuracy scores. Within the HIV-SP group, WTAR scores were negatively correlated with the total number of errors for the Anger videos, $r(49)=-.48, p<.000$. Additionally, WTAR scores were negatively correlated with the total number of errors across all of the videos, $r(49)=-.34$, $p<.02$. The findings indicate that higher intellectual function was associated with fewer errors. Average latency was not correlated with WTAR scores, either between or within groups, for Sad, Fear, Surprise, Disgust, Anger, or Happiness videos.

\subsection{Relationship between physiological baselines and performance on SCAN and DARE}

Correlational analyses were conducted, between and within groups, to determine the relationship between initial baseline physiological measures and performance on the SCAN and DARE.

3.5.1 SCAN-When collapsed across groups, performance on the Filtered Words subtest was significantly correlated to initial baseline RSA, $r(79)=.28, p<.01$ (i.e., higher RSA related to better performance on the Filtered Words subtest). None of the initial baseline physiological measures were related to performance on the Competing Words subtest.

\subsubsection{DARE}

3.5.2.1 Accuracy: Within the HIV-SN group only, the total number of errors on the Fear videos was significantly negatively correlated to initial baseline heart rate, $r(20)=-.54, p<$. 01 (i.e., higher heart rate related to fewer errors).

3.5.2.2 Latency: The average latency for the Sad videos was significantly negatively correlated to initial baseline heart rate within the HIV-SP group only, $r(54)=-.32, p<.02$ (i.e., higher heart rate related to shorter latencies). The average latency for the Surprise videos was significantly negatively correlated to initial baseline RSA within the HIV-SN group only, $r(20)=-.49, p<.03$ (i.e., higher RSA related to shorter latencies).

\subsection{HIV-related analyses}

3.6.1 Viral load-Viral load was not a significant covariate in any physiological model nor was it consistently correlated with accuracy of /latency in detecting emotions, auditory processing measures, or any baseline physiological variable.

3.6.2 CD4-CD4 was negatively correlated with total number of errors on the DARE, I(76) $=-.30, p<.009$ (i.e., higher $\mathrm{CD} 4$ associated with fewer errors). $\mathrm{CD} 4$ was not correlated with any of the cardiopulmonary or auditory processing measures.

3.6.3 Duration of HIV infection-Duration of known HIV infection was not a significant covariate in any physiological model. It was not consistently correlated with accuracy of / latency in detecting emotions, auditory processing measures, or any baseline physiological variable. Duration of known HIV infection was not correlated with any of the initial physiological baselines, or performance on SCAN or DARE. 


\section{Discussion}

The study was theoretically driven by the integrated Social Engagement System proposed in the Polyvagal Theory (Porges, 1995, 2007, 2009). The study provided an opportunity to evaluate the covariation of visceromotor and somatomotor components as a function of HIV infection. The visceromotor component was assessed by measuring the output of the central regulation of myelinated vagus to the sinoatrial node (i.e., RSA). The somatomotor component was assessed by measuring variables dependent on cranial nerve regulation of the striated muscles of the face and head involved in auditory processing (i.e., via neural regulation of the middle ear muscles) and gaze required for affect awareness (i.e., via the neural regulation of the orbicularis oculi). It was hypothesized that HIV infection would depress both the visceromotor (i.e., autonomic function) and somatomotor (i.e., affect recognition and auditory processing) components. The data supported this hypothesis. HIVseropositive women had reduced RSA and poorer performance on the affect recognition and dichotic listening tests. Additionally, within the HIV-seropositive group, accuracy on the affect recognition task was correlated with markers of HIV disease progression.

Individuals in the HIV-SP group had lower baseline RSA, although baseline heart rate was similar between groups. Reduced RSA indicates a reduction of myelinated vagal control of the heart (i.e., release of the vagal brake), which is necessary for a coordinated cardiovascular response that will result in the increased heart rate required during exercise (e.g., Hatfield et al., 1998) and often experienced during states of anxiety (Pittig et al., 2013).

The group difference in RSA indicates reduced myelinated vagal influence to the heart in women who are HIV- seropositive, suggesting that these individuals may be in a physiological state that supports anxious and mobilization behaviors, such as fight/flight, rather than a physiological state that supports social engagement. Reduced vagal influence to the heart not only affects state regulation, but is an essential component of the Social Engagement System that involves the neural regulation of the muscles of the face and head (Porges, 2007, 2009).

A lack of group differences in heart rate indicates a possible activation of compensatory extra-vagal mechanisms, such as neuroendocrine feedback loops and/or sympathetic activity, which ultimately resulted in equivalent heart rate between groups. While these compensatory mechanisms were not assessed in the current study, future research could assess these hypothetical mechanisms by including such tasks as cold pressor assessment, and/or posture shift to assess baroreceptor response. Sympathetic activity could also be assessed in future studies, particularly since researchers have reported that autonomic nervous system activity (a composite variable consisting of skin conductance, brachial artery systolic blood pressure, interbeat-interval, finger photoplethysmograph pulse peak amplitude, and peripheral pulse transit time) mediated the relationship between social inhibition and virologic indicators of disease progression in men with HIV infection (Cole et al., 2003) and affected response to HAART treatment (Cole et al., 2001). A study evaluating both parasympathetic activity and sympathetic activity, as described in the aforementioned studies, and neuroendocrine variables (e.g., cortisol, vasopressin, oxytocin) could provide a more complete link between autonomic nervous system activity, social behavior, and HIV disease progression.

The HIV- seropositive group performed significantly worse than the HIV- seronegative group on the dichotic listening and affect recognition tests. The dichotic listening task required participants to repeat the word spoken in either the left or right ear, when two different words were presented to each ear simultaneously. The results demonstrate that 
individuals who are HIV-seropositive may have more difficulty focusing on a conversation in environments in which several people are talking simultaneously. Dichotic listening tasks, such as competing words, have been used as a proxy for central cholinergic function (Drachman et al., 1980) and in the current study performance on the competing words test was significantly correlated with WTAR within the HIV-SP group. Moreover, there was a trend towards poorer WTAR performance in the HIV-SP group consistent with the reports in the literature on cognitive deficits associated with HIV and the literature on dampened central cholinergic function and cognitive deficits.

Since vagal efferent influences on the heart and middle ear muscle tone are peripheral measures and dependent on both central and peripheral influences, the correlation between Filtered Words and RSA across all participants is consistent with predictions based on the Polyvagal Theory (see Porges and Lewis, 2009), which links cardiac vagal tone (i.e., low RSA) with a low neural tone to the middle ear muscles (Lewis et al., 2011). The low middle ear muscle tone would result in the "transfer function" of middle ear structures passing more low frequency acoustic energy into the inner ear and functionally masking the higher frequency components of speech necessary to extract human voice in noisy environments. Given the ambient noise in everyday life and the high risk nature of all participants in this study, independent of serostatus, many of the participants would have difficulties in attending to and accurately understanding speech. This difficulty in extracting voice from background sounds could greatly impact social behavior, increase frustration in social settings, and degrade the effectiveness of social-support seeking behaviors.

It is important to note that although RSA, across all participants, was significantly correlated with performance on the Filtered Words test, Filtered Words performance was not significantly degraded in the HIV-SP relative to the HIV-SN group. In contrast, performance on the Competing Words test was significantly degraded in the HIV-SP group, but was not correlated with RSA. There are several relevant points related to this observation. First, the groups of participants are defined by great diversity in clinical history, general health, cognitive function, and adaptive social behavior. The heterogeneity of autonomic features and cognitive function may have contributed to an experimental design that was underpowered to detect HIV-related effects on the Filtered Words test. Thus, as illustrated in Figure 1, the trend towards group differences could not be detected with the current experimental design.

Second, since the correlation between scores on the two auditory processing tasks was approximately .3 within each group and across both groups, the two tasks are indexing relatively independent processes. For example, the Filtered Words task may be more dependent on the peripheral neural regulation of the autonomic nervous system (i.e., RSA) and of the middle ear structures. In contrast, the Competing Words task may be more dependent on central cholinergic pathways involved in cognitive processes. Based on our "cholinergic hypothesis" (see below), we assume that the HIV virus has effects on different levels of the nervous system. The group differences in the Competing Words test may reflect a combined effect dampening both central and peripheral cholinergic pathways, which would make the task less sensitive to vagal regulation of the heart (i.e. RSA), while the Filtered Words test may be a more sensitive indicator of a degrading of peripheral cholinergic function manifested in RSA.

The affect recognition test required participants to identify the specific emotion illustrated in each stimulus movie. Individuals, who were HIV- seropositive, made significantly more errors in the identification of specific emotions. Within the HIV- seropositive group only, intellectual functioning further impacted affected accuracy scores, as participants with higher intellectual function made fewer errors. Additionally, individuals who were HIV- 
seropositive did not demonstrate a relationship between accuracy and latency. These findings suggest that individuals who are HIV- seropositive may have difficulty interpreting facial features of emotion (i.e., during a face-to-face conversation), that could possibly be due, in part, to reduced intellectual functioning, which could ultimately lead to the misinterpretation of emotional cues and trigger negative reactions in others.

By demonstrating that depressed vagal regulation of the heart rate manifested in RSA covaries within the HIV- seropositive group with poorer affect recognition and auditory processing, the data support the proposition of an integrated Social Engagement System with common neural mechanisms and common neural vulnerabilities. These findings are consistent with recent reports in which individuals with autism spectrum disorder, a clinical disorder associated with depressed RSA, also had compromised auditory processing skills (Porges et al., 2012) and compromised affect recognition (Bal et al., 2009).

\subsection{Future directions}

4.1.1 The Cholinergic Hypothesis-Data from the current study suggest that the HIV has an impact on the cholinergic system. This was evidenced by lower RSA and poorer performance on the dichotic listening and affect recognition tasks. RSA provides an index of cholinergic activity in the periphery, since acetylcholine is the neurotransmitter that mediates the influence of the myelinated vagus on the heart. In support of this conclusion, it has been reported that HIV compromises parasympathetic functioning (Lebach et al., 2007; Mittal et al., 2004). Other research has provided a link between central cholinergic function and dichotic listening and affect recognition. Consistent with this general "cholinergic" deficit hypothesis, dichotic listening and affect recognition are also impaired by anticholinergic blockades such as scopolamine (dichotic listening: Drachman et al., 1980; affect recognition: Kamboj and Curran, 2006). The current study and the Kamboj and Curran (2006) study consistently reported deficits in accurately detecting negative emotions, although the specific emotions that were affected (Fear in the current study, Sadness and Anger in the Kamboj and Curran study) may have been due to the qualitative differences between the stimuli in the two studies. Since deficits in dichotic listening, affect recognition, and vagal regulation of the heart can be mimicked by anticholinergic manipulations, the results suggest that HIV may compromise behavioral and autonomic function through an anticholinergic mechanism. In summary, other laboratories, consistent with our preliminary findings, have reported deficits in autonomic functioning, dichotic listening, and recognition of human facial expressions consistent with the cholinergic burden of disease.

In conclusion, the present study demonstrated that autonomic and behavioral features of the Social Engagement System are depressed in HIV-infected women. Future studies, guided by the Cholinergic Hypothesis and the Polyvagal Theory, could evaluate additional measures dependent on the Social Engagement System to expand on these findings.

The current study focused on female participants only. Although there may be gender differences in baseline physiological state and/or affect recognition, the effect of HIV serostatus in males may be of interest for future studies, which could then evaluate the generalizability of the current findings to other adults who are HIV-seropositive.

Longitudinal studies could be designed to examine the covariation of autonomic and behavioral features with HIV disease progression. For example, in the current study, the HIV-seropositive group reported significantly more depressive symptoms than the HIVseronegative group (see Table 1), but the CESD scores did not function as significant covariates in the autonomic, auditory processing or affect recognition analyses presented in this paper (analyses not reported, as these results were likely due to a lack of statistical power). However, given the extensive literature demonstrating the impact of depression on 
autonomic functioning (e.g., Rottenberg, 2007) and the high correlation between HIV serostatus and depression (e.g., Ownby et al., 2010) future studies should evaluate the inter relatedness of depression, HIV disease progression, and autonomic and behavioral indices in a larger sample size. Although thirty-one participants in the HIV- seropositive group met diagnostic criteria for AIDS, the present database was insufficiently powered to analyze for group differences in AIDS status within the HIV-seropositive group. Future studies could include a larger sample to evaluate the effect of AIDS status on the autonomic and social/ behavioral variables of interest. In addition, research could be expanded to investigate the specific nature of the debilitating effect of HIV by evaluating such potential mediating factors as aging/menopause, inflammation, immune senescence, and other medical and psychiatric comorbidities including substance abuse, trauma/PTSD, and psychosocial and environmental stressors.

\section{Acknowledgments}

The data described in this manuscript were collected by the Chicago site of the Women's Interagency HIV study (WIHS), which is funded by the National Institute of Allergy and Infectious Diseases Grant U01-AI-34994 (PI, Dr. Mardge Cohen) and co-funded by the National Cancer Institute and the National Institute of Drug Abuse. Kathleen Weber is funded, in part, by P30- AI 082151. The research was supported, in part, by NIH research grant MH060625 awarded to S.W. Porges. K.J. Heilman was supported, in part, by the Biomedical Neuroscience Training Program T32 MH067631. The contents of this publication are solely the responsibility of the authors and do not necessarily represent the views of the National Institutes of Health.

\section{References}

Amayo EO, Kwasa TO. HIV and acute peripheral facial nerve palsy. East African Medical Journal. 1991; 68:948-951. [PubMed: 1800092]

Applebaum AJ, Bullis JR, Traeger LN, O'Cleirigh C, Otto MW, Pollack MH, Safren SA. Rates of mood and anxiety disorders and contributors to continued heroin use in methadone maintenance patients: A comparison by HIVstatus. Neurobehavioral HIV Medicine. 2010; 2:49-57.

Bacon MC, Von Wyl V, Alden C, Sharp G, Robison E, Hessol N, Gange S, Barranday Y, Holman S, Weber K, Young MA. The Women's Interagency HIV Study: An observational cohort brings clinical sciences to the bench. Clinical and Diagnostic Laboratory Immunology. 2005; 12(9):10131019. [PubMed: 16148165]

Bal E, Harden E, Lamb D, Van Hecke AV, Denver JW, Porges SW. Emotion recognition in children with autism spectrum disorders: Relations to eye gaze and autonomic state. Journal of Autism and Developmental Disorders. 2010; 40:358-370. [PubMed: 19885725]

Barkan SE, Melnick SL, Preston-Martin S, Weber K, Kalish LA, Miotti P, Young M, Greenblatt R, Sacks H, Feldman J. The Women's Interagency HIV Study. Epidemiology. 1998; 9(2):117-125. [PubMed: 9504278]

Brownley KA, Milanovich JR, Motivala SJ, Schneiderman N, Fillion L, Graves JA, Klimas N, Fletcher MA, Hurwitz BE. Autonomic and cardiovascular function in HIVspectrum disease: Early indicators of cardiac pathophysiology. Clinical Autonomic Research. 2001; 11:319-326. [PubMed: 11758799]

Cade WT, Reeds DN, Lassa-Claxton S, Davila-Roman VG, Waggoner AD, Powderly WG, Yarasheski KE. Post-exercise heart rate recovery in HIV-positive individuals on highly active antiretroviral therapy. Early indicator of cardiovascular disease? HIV Medicine. 2008; 9:96-100. [PubMed: 18093131]

Calkins SD, Graziano PA, Keane SP. Cardiac vagal regulation differentiates among children at risk for behavior problems. Biological Psychology. 2007; 74(2):144-53. [PubMed: 17055141]

Ciesla JA, Roberts JE. Meta-analysis of the relationship between HIV infection and risk for depressive disorders. American Journal of Psychiatry. 2001; 158:725-730. [PubMed: 11329393]

Cohn JF, Zlochower AJ, Lien J, Kanade T. Automated face analysis by feature point tracking has high concurrent validity with manual FACS coding. Psychophysiology. 1999; 36(1):35-43. [PubMed: 10098378] 
Cole SR, Hernan MA, Anastos K, Jamieson BD, Robins JM. Determining the effect of highly active antiretroviral therapy on changes in human immunodeficiency virus type 1 RSA viral load using a marginal structural left-censored mean model. American Journal of Epidemiology. 2007; 166(2): 219-227. [PubMed: 17478436]

Cole SW, Kemeny ME, Fahey JL, Zack JA, Naliboff BD. Psychological risk factors for HIV pathogenesis: Mediation by the autonomic nervous system. Biological Psychiatry. 2003; 54:14441456. [PubMed: 14675810]

Cole SW, Naliboff BD, Kemeny ME, Griswold MP, Fahey JL, Zack JA. Impaired response to HAART in HIV-infected individuals with high autonomic nervous system activity. Proceedings of the National Academy of Sciences. 2001; 98(2):12695-12700.

Compostella C, Compostella L, D'Elia R. Cardiovascular autonomic neuropathy in HIV-positive African patients. Minerva Cardioangiologica. 2008; 56(4):417-28. [PubMed: 18614986]

Correia D, Rodrigues de Resende LAP, Molina RJ, Ferreira BD, Colombari F, Barbosa CJ, Da Silva VJ, Prata A. Power spectral analysis of heart rate variability in HIV-infected and AIDS patients. Pacing and Clinical Electrophysiology. 2006; 29:53-58. [PubMed: 16441718]

Daluwatte C, Miles JH, Christ SE, Beversdorf DQ, Takahashi TN, Yao G. Atypical pupillary light reflex and heart rate variability in children with autism spectrum disorder. Journal of Autism and Developmental Disabilities (Epub). 2012

Drachman DA, Noffsinger D, Sahakian BJ, Kurdziel S, Fleming P. Aging, memory and the cholinergic system: A study of dichotic listening. Neurobiology Aging. 1980; 1:38-43.

Hall SS, Lightbody AA, Huffman LC, Lazzeroni LC, Reiss AL. Physiological correlates of social avoidance behavior in children and adolescents with fragile $\mathrm{X}$ syndrome. Journal of the American Academy of Child and Adolescent Psychiatry. 2009; 48(3):320-9. [PubMed: 19182690]

Hatfield BD, Santa Maria DL, Porges SW, Potts JT, Spalding T, Byrne EA. Respiratory sinus arrhythmia during exercise in aerobically trained and untrained men. Medicine and Science in Sports and Exercise. 1998; 30:206-214. [PubMed: 9502347]

Heilman KJ, Porges SW. Accuracy of the LifeShirt (Vivometrics) in the detection of cardiac rhythms. Biological Psychology. 2007; 75(3):300-5. [PubMed: 17540493]

Hessol NA, Weber KM, Holman S, Robison E, Goparaju L, Alden CB, Kono N, Watts DH, Ameli N. Retention and attendance of women enrolled in a large prospective study of HIV-1 in the United States. Journal of Women's Health. 2009; 18(10):1627-1637.

Kamboj SK, Curran HV. Scopolamine induces impairments in the recognition of human facial expressions of anger and disgust. Psychopharmacology. 2006; 185:529-535. [PubMed: 16555061]

Keith, RW. SCAN: A screening test for auditory processing disorders. The Psychological Corporation; San Antonio, TX: 1986.

Komolafe MA, Fatusi OA, Alatise OI, Komolafe EO, Amusa YB, Adeolu AA, Durosinmi MA. The role of human immunodeficiency virus infection in infranuclear facial paralysis. Journal of the National Medical Association. 2009; 101:361-366. [PubMed: 19397228]

Lebach A, Kristoffersen US, Mehlsen J, Wiinberg N, Petersen CL, Hesse B, Gerstoft J, Kjaer A. Autonomic dysfunction in HIV patients on antiretroviral therapy: Studies of heart rate variability. Clinical Physiology and Functional Imaging. 2007; 27:363-367. [PubMed: 17944658]

Lewis GF, Furman SF, McCool MF, Porges SW. Statistical strategies to quantify respiratory sinus arrhythmia: Are commonly used metrics equivalent? Biological Psychology. 2012; 89:349-364. [PubMed: 22138367]

Lewis GF, Stanfill S, Zageris D, Davila M, Porges SW. Neural regulation of sensory gating in the auditory periphery: Relationship with listening and heart rate dynamics. Psychophysiology. 2011; 48(Suppl. 1):S96-S96.

Lyon DE, Munro C. Disease severity and symptoms of depression in Black Americans infected with HIV. Applied Nursing Research. 2001; 13:3-10. [PubMed: 11172224]

Martin-Ruiz C, Court J, Lee M, Piggott M, Johnson M, Ballard C, Kalaria R, Perry R, Perry E. Nicotinic receptors in dementia of Alzheimer, Lewy body and vascular types. Acta Neurologica Scandinavica Supplementum. 2000; 176:34-41. [PubMed: 11261803]

Marubio LM, Paylor R. Impaired passive avoidance learning in mice lacking central neuronal nicotinic acetylcholine receptors. Neuroscience. 2004; 129:575-582. [PubMed: 15541879] 
Mittal CM, Wig N, Mishra S, Deepak KK. Heart rate variability in human immunodeficiency viruspositive individuals. International Journal of Cardiology. 2004; 94:1-6. [PubMed: 14996467]

Ownby RL, Jacobs RJ, Waldrop-Valverde D, Gould F. Depression care and prevalence in HIVpositive individuals. Neurobehavioral HIV Medicine. 2010; 2:73-83.

Patriquin MA, Scarpa A, Friedman BH, Porges SW. Respiratory sinus arrhythmia: A marker for positive social functioning and receptive language skills in children with autism spectrum disorders. Developmental Psychobiology. 2013; 55:101-112. [PubMed: 22212893]

Pence BW, Miller WC, Whetten K, Eron JJ, Gaynes BN. Prevalence of DSM-IV-defined mood, anxiety, and substance use disorders in an HIV clinic in the Southeastern United States. Journal of Acquired Immune Deficiency. 2006; 42(3):298-306.

Pittig A, Arch JJ, Lam CWR, Craske MG. Heart rate and heart rate variability in panic, social anxiety, obsessive-compulsive, and generalized anxiety disorders at baseline and in response to relaxation and hyperventilation. International Journal of Psychophysiology. 2013; 87(1):19-27. [PubMed: 23107994]

Porges, SW. U.S. Patent Office; Washington DC: 1985. Method and Apparatus for Evaluating Rhythmic Oscillations in Aperiodic Physiological Response Systems.. Patent Number: 4,510,944

Porges SW. Orienting in a defensive world: Mammalian modifications of our evolutionary heritage. A Polyvagal Theory. Psychophysiology. 1995; 32:301-318. [PubMed: 7652107]

Porges SW. Social engagement and attachment: A phylogenetic perspective. Roots of Mental Illness in Children. Annals of the New York Academy of Sciences. 2003; 1008:31-47.

Porges SW. Neuroception: A subconscious system for detecting threat and safety. Zero to Three: Bulletin of the National Center for Clinical Infant Programs. 2004; 24(5):19-24.

Porges SW. The polyvagal perspective. Biological Psychology. 2007; 74:116-43. [PubMed: 17049418]

Porges SW. The polyvagal theory: New insights into adaptive reactions of the autonomic nervous system. Cleveland Clinic Journal of Medicine. 2009; 76(Suppl 2):S86-90. [PubMed: 19376991]

Porges, SW. The Polyvagal Theory: Neurophysiological Foundations of Emotions, Attachment, Communication, and Self-regulation. WW Norton; New York: 2011.

Porges SW, Furman SA. The early development of the autonomic nervous system provides a neural platform for social behavior: A polyvagal perspective. Infant and Child Development. 2011; 20(1): 106-118. [PubMed: 21516219]

Porges, SW.; Bohrer, RE. Analyses of periodic processes in psychophysiological research.. In: Cacioppo, JT.; Tassinary, LG., editors. Principles of Psychophysiology: Physical, Social, and Inferential Elements. Cambridge University Press; New York: 1990. p. 708-753.

Porges SW, Byrne EA. Research methods for measurement of heart rate and respiration. Biological Psychology. 1992; 34(2-3):93-130. [PubMed: 1467397]

Porges, SW.; Lewis, GF. The polyvagal hypothesis: Common mechanisms mediating autonomic regulation, vocalizations, and listening.. In: Brudzynski, SM., editor. Handbook of Mammalian Vocalizations: An Integrative Neuroscience Approach. Academic Press; Amsterdam: 2009. p. 255-264.

Porges, SW.; Cohn, JF.; Bal, E.; Lamb, D. The Dynamic Affect Recognition Evaluation, Version 1. Brain-Body Center. University of Illinois at Chicago; Chicago, IL: 2007.

Porges SW, Macellaio M, Stanfill SD, McCue K, Lewis GF, Harden ER, Handelman M, Denver J, Bazhenova OV, Heilman KJ. Respiratory sinus arrhythmia and auditory processing in autism: Modifiable deficits of an integrated social engagement system? International Journal of Psychophysiology Epub. 2012

Prachakul W, Grant JS, Keltner NL. Relationships among functional social support, HIV-related stigma, social problem solving, and depressive symptoms in people living with HIV: a pilot study. Journal of the Association of Nurses in AIDS Care. 2007; 18(6):67-76. [PubMed: 17991600]

Radloff LS. The CES-D scale: a self-report depression scale for research in the general population. Applied Psychological Measurement. 1977; 1:385-401.

Riniolo T, Porges SW. Inferential and descriptive influences on measures of respiratory sinus arrhythmia: Sampling rate, R-wave trigger accuracy and variance estimates. Psychophysiology. 1997; 34(5):613-21. [PubMed: 9299916] 
Roberts JE, Boccia ML, Bailey DB Jr. Hatton DD, Skinner M. Cardiovascular indices of physiological arousal in boys with fragile X syndrome. Developmental Psychobiology. 2001; 39(2):107-23. [PubMed: 11568881]

Robertson KR, Smurzynski M, Parsons TD, Wu K, Bosch RJ, Wu J, McArthur JC, Collier AC, Evans SR, Ellis RJ. The prevalence and incidence of neurocognitive impairment in the HAART era. AIDS. 2007; 21(14):1915-1921. [PubMed: 17721099]

Rottenberg J. Cardiac vagal control in depression: a critical analysis. Biological Psychology. 2007; 74:200-11. [PubMed: 17045728]

Sakhuja A, Goval A, Jarval AK, Wig N, Vajpayee M, Kumar A, Deepak KK. Heart rate variability and autonomic function tests in HIV positive individuals in India. Clinical Autonomic Research. 2007; 17(3):193-6. [PubMed: 17390100]

Schrimshaw EW. Relationship-specific unsupportive social interactions and depressive symptoms among women living with HIV/AIDS: Direct and moderating effects. Journal of Behavioral Medicine. 2003; 26:297-313. [PubMed: 12926407]

Simpson DM, Tagliati M. Neurological manifestations of HIV infection. Annals of Internal Medicine. 1994; 121:769-785. [PubMed: 7794311]

Weber E, Blackstone K, Woods SP. Cognitive neurorehabilitation of HIV-associated neurocognitive disorders: A qualitative review and call to action. Neuropsychology review. 2013; 23(1):81-98. [PubMed: 23417497]

Wechsler, D. Wechsler test of adult reading. The Psychological Corporation; San Antonio, TX: 2001. 


\section{Highlights}

HIV-infected women had less heart rate variability (i.e., RSA)

HIV-infected women had poorer performance on auditory processing task

HIV-infected women had poorer performance on affect recognition task

There was a negative correlation between CD4 and accuracy to detect specific emotions 


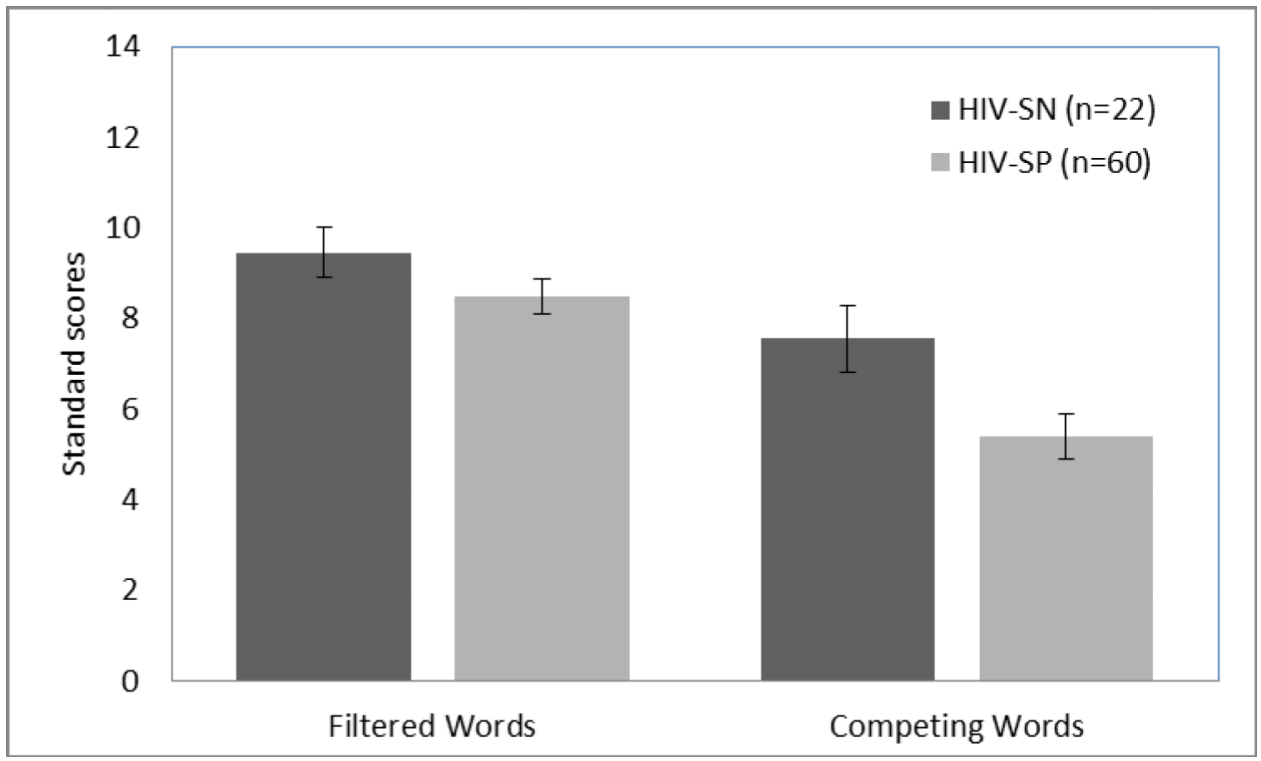

Figure 1.

Significant group differences in Competing Words (SE bars), but non-significant differences in Filtered Words. 
Table 1

Socio-demographic and clinical characteristics for each HIV serostatus group.

\begin{tabular}{|c|c|c|}
\hline & HIV-SP $(n=61)$ & HIV-SN $(n=22)$ \\
\hline $\begin{array}{l}\text { Age (years) } \\
{\left[A N O V A, F(1,82)=3.60, p<.06, \eta \rho^{2}=.04, \text { observed power }=.47\right]}\end{array}$ & $\begin{array}{l}\mathrm{M}(\mathrm{SD})=42.16(8.1) \\
\text { Range }=27-61 \text { years }\end{array}$ & $\begin{array}{l}\mathrm{M}(\mathrm{SD})=38.09(10.01) \\
\text { Range }=24-54 \text { years }\end{array}$ \\
\hline $\begin{array}{l}\text { Highest education } \\
\text { Did not complete high school } \\
\text { Completed high school } \\
\text { Some college/college degree/advanced degree } \\
\text { Did not report education } \\
\text { (Independent samples, Mann-Whitney U test, } p<.81 \text { ) }\end{array}$ & $\begin{array}{l}n=19 \\
n=22 \\
n=19 \\
n=1\end{array}$ & $\begin{array}{l}n=7 \\
n=7 \\
n=7 \\
n=1\end{array}$ \\
\hline $\begin{array}{l}\text { Racial/Ethnic group } \\
\text { White } \\
\text { African-American } \\
\text { Latino (non-African American) } \\
\text { Other } \\
\text { Did not report } \\
\left.\text { [Pearson Chi-Square (exact), } X^{2}(4)=1.67, p<.91\right]\end{array}$ & $\begin{array}{l}n=3 \\
n=51 \\
n=4 \\
n=1 \\
n=2\end{array}$ & $\begin{array}{l}n=0 \\
n=19 \\
n=2 \\
n=0 \\
n=1\end{array}$ \\
\hline $\begin{array}{l}\text { Household income } \\
\text { Less than } \$ 12,000 \\
\text { More than } \$ 12,001 \\
\text { (Independent samples, Mann-Whitney U test, } p<.09 \text { ) }\end{array}$ & $\begin{array}{l}n=38 \\
n=23\end{array}$ & $\begin{array}{l}n=9 \\
n=13\end{array}$ \\
\hline 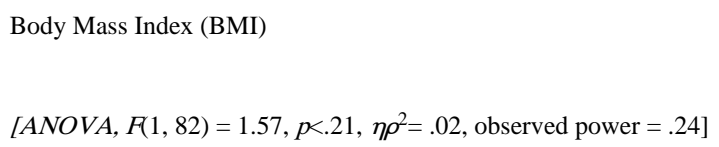 & $\begin{array}{l}\mathrm{M}(\mathrm{SD})=30.60(8.73) \\
\text { Range } 20.80-57.30\end{array}$ & $\begin{array}{l}\mathrm{M}(\mathrm{SD})=28.07(7.90) \\
\text { Range } 16.10-49.80\end{array}$ \\
\hline $\begin{array}{l}\text { WTAR scores } \\
{\left[A N O V A, F(1,75)=3.02, p^{<.09}, \eta \rho^{2}=.04, \text { observed power }=.40\right]}\end{array}$ & $\mathrm{M}(\mathrm{SD})=22.67(10.79), n=54$ & $\mathrm{M}(\mathrm{SD})=27.27(9.65), n=22$ \\
\hline 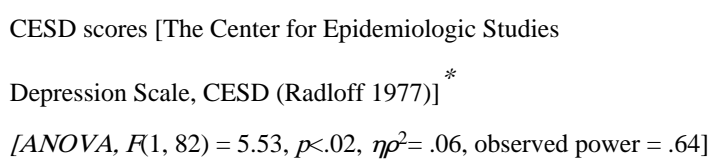 & $\mathrm{M}(\mathrm{SD})=15.66(10.47), n=61$ & $\mathrm{M}(\mathrm{SD})=9.77$ (8.77), $n=22$ \\
\hline $\begin{array}{l}\text { Smoking patterns at time of testing } \\
\text { [Pearson Chi Square, } X^{2}(1)=.48, p<.49 \text { ] }\end{array}$ & $\begin{array}{l}\text { Not smoking: } n=33 \\
\text { Currently smoking: } n=28\end{array}$ & $\begin{array}{l}\text { Not smoking: } n=10 \\
\text { Currently smoking: } n=12\end{array}$ \\
\hline Average alcohol use at time of testing & $\begin{array}{l}\text { Abstaining: } n=41 \\
\text { Light ( }<3 \text { drinks/week): } n=12 \\
\text { Moderate (3-13 drinks/week): } n=5\end{array}$ & $\begin{array}{l}\text { Abstaining: } n=9 \\
\text { Light ( }<3 \text { drinks/week): } n=11 \\
\text { Moderate (3-13 drinks/week): } n=1\end{array}$ \\
\hline
\end{tabular}




\begin{tabular}{|c|c|c|}
\hline & HIV-SP $(n=61)$ & HIV-SN $(n=22)$ \\
\hline (Independent samples, Mann-Whitney $U$ test, $p<.09$ ) & Heavier (>=14 drinks/week): $n=3$ & Heavier (>=14 drinks/week): $n=1$ \\
\hline $\begin{array}{l}\text { History of violence exposure } \\
\text { [Pearson Chi Square, } X^{2}(1)=3.16, p<.08 \text { ] }\end{array}$ & $\begin{array}{l}\text { Past violence reported: } n=29 \\
\text { Never/no violence: } n=18 \\
\text { Missing: } n=14\end{array}$ & $\begin{array}{l}\text { Past violence reported: } n=16 \\
\text { Never/no violence: } n=3 \\
\text { Missing: } n=3\end{array}$ \\
\hline Nadir CD4 distribution $(n=55)$ & $\begin{array}{l}\mathrm{M}(\mathrm{SD})=291.33(168.57) \\
\text { Range 3-736 }\end{array}$ & \\
\hline $\mathrm{CD} 4$ at time of research session $(n=61)$ & $\begin{array}{l}\mathrm{M}(\mathrm{SD})=437.56(321.29) \\
\text { Range 2-1684 } \\
<200: n=12 \\
\text { 200-349: } n=17 \\
\text { 350-499: } n=13 \\
>500: n=19\end{array}$ & \\
\hline Viral load $[(\log 10(\mathrm{VL})]$ at time of research session $(n=61)$ & $\begin{array}{l}\mathrm{M}(\mathrm{SD})=3.07(1.31) \\
\text { Range 1.68-6.28 }\end{array}$ & \\
\hline ARV adherence & $\begin{array}{l}<95 \% \text { compliance: } n=15 \\
95 \% \text { or higher compliance: } n=32 \\
\text { Not applicable } n=13 \\
\text { Data not available } n=1\end{array}$ & \\
\hline Duration of HIV at time of research session & $\begin{array}{l}23.41 \text { years: } n=1 \\
15-19.99 \text { years: } n=10 \\
10-14.99 \text { years: } n=27 \\
5-9.99 \text { years: } n=16 \\
4-4.99 \text { years: } n=7\end{array}$ & \\
\hline Duration of AIDS at time of research session & $\begin{array}{l}10-14.08 \text { years: } n=12 \\
5-9.99 \text { years: } n=3 \\
1.54-4.99 \text { years: } n=13\end{array}$ & \\
\hline
\end{tabular}

* group differences were significant at $p<.05$ 
Table 2

Descriptive statistics of physiological variables for each HIV serostatus group.

\begin{tabular}{llll}
\hline & RSA (sd) & HR (sd) & Respiration Rate (sd) \\
\hline Initial baseline analyses only & & & \\
HIV-SN (n=20) & $5.91(1.62)$ & $76.02(12.70)$ & $18.25(3.73)$ \\
HIV-SP (n=60) & $4.95(1.56)$ & $77.72(10.64)$ & $17.69(3.51)$ \\
Repeated measures analyses Initial baseline & & & \\
HIV-SN (n=19) & $5.98(1.63)$ & $75.29(12.62)$ & $18.58(3.52)$ \\
HIV-SP (n=50) & $5.05(1.60)$ & $77.55(11.03)$ & $17.33(3.52)$ \\
Pre-DARE baseline & & & \\
HIV-SN (n=19) & $6.12(1.27)$ & $70.96(11.50)$ & $18.87(3.71)$ \\
HIV-SP (n=50) & $5.53(1.67)$ & $73.03(10.25)$ & $16.91(3.40)$ \\
Post-DARE baseline & & & \\
HIV-SN (n=19) & $6.20(1.42)$ & $70.32(11.65)$ & $19.23(4.19)$ \\
HIV-SP (n=50) & $5.50(1.62)$ & $72.88(9.98)$ & $16.80(3.61)$
\end{tabular}

